\title{
Pedestrian Protection System for ADAS using ARM 9
}

\author{
Rajashri Sanatkumar Dixit \\ E\&TC Department \\ SITRC, Sandip Foundatrion \\ Nashik, Maharashtra, India
}

\author{
S.T. Gandhe \\ E\&TC Department \\ SITRC, Sandip Foundatrion \\ Nashik, Maharashtra, India
}

\author{
Pravin Dhulekar \\ E\&TC Department \\ SITRC, Sandip Foundatrion \\ Nashik, Maharashtra, India
}

\begin{abstract}
We developed pedestrian protection system by using haar cascade algorithm with Friendly ARM (S3C2440) board as a hardware. This system is developed on opencv platform using ubuntu as an operating system. This system will work in two mode auto and manual. In auto mode as soon as pedestrian get detected break will be applied if pedestrian is in high risk area. And for manual mode there is alarm or buzzer will sound to alert driver. This system gives low false positive as well as low false negative rate. This system is low cost system as compare with state of art.
\end{abstract}

\section{General Terms}

Computer vision, Machine intelligence.

\section{Keywords}

Feature extraction, friendly ARM, haar cascade algorithm, Pedestrian detection.

\section{INTRODUCTION}

Pedestrian detection system is having applications in the field of surveillance, robotics, advanced driver assistance system (ADAS) etc. Advanced driver assistance systems are basically developed to help driver while driving, in the form of warning drivers, providing better visibility etc. so as to minimize human errors which causes serious issues like accidents, pedestrian protection systems helps in the same way. Pedestrian protection system takes input images of stationary as well as moving people in the path of car using camera, in real time. And then warn driver accordingly if there are chances of collision in manual driving car. If car is in autopilot mode then accordingly break will get apply and necessary action will be taken. Researchers are facing the challenge of increasing computational speed, increasing robustness of system and maximizing accuracy.

The intelligent vehicles which use pedestrian protection techniques are based on feature extraction and also classification. Several techniques have formerly been proposed for pedestrian detection. . Viola and Jones [3][4] introduced integral image concept, Also they used adaboost classifier with cascading of classifiers. Navneet Dalal [5] proposed HOG and SVM based method for pedestrian detection where HOG is feature extractor and SVM is classifier. Results are tested on two datasets MIT dataset and INRIA dataset. Xianbin Cao et al. [8] proposed method for pedestrian detection in unseen scenes. Swift adaptive method for pedestrian detection which is based on cascade classifier along with ternary pattern is proposed. Jiang-tao Wang et al. [9] proposed method for pedestrian detection and also tracking in infrared images. GMM background model is firstly apply for separation of foreground candidates from background, then a shape describer is used for constructing the feature vector for pedestrian, then SVM is used for training the data. After that a multi-cues fusing algorithm is applied. Lie Guo et al. [10] proposed two stage pedestrian detection method. In first stage Adaboost algorithm as well as cascade algorithm is used and in second stage to confirm presence of pedestrian SVM classifier is used. Hulin Kuang et al. [11] used MutualCascade method for pedestrian detection. This mutual cascade method consists of Gentle Adaboost (GAB) cascade as well as the Four Direction Feature (FDF). To accomplish robustness in addition to minimize ambiguity in human detection, in past few years incredible research efforts have been done [6][7].

In this paper pedestrian protected system using friendly ARM board S3C2440 with haar cascade algorithm is proposed. In this system pedestrian get detected just as they enter the camera range, with low false alarm rate and high speed. As pedestrian get detected speed of vehicle get reduced i.e. break get applied in automatic cars or vehicles but in case of manual cars alarm will blow to alert driver and also deploy external airbags if a collision is unavoidable. Workflow of pedestrian protection system includes preprocessing, feature extraction, classification, verification, tracking (for video), and application, as shown in fig. 1 .

Feature extraction is necessary to identify targeted object. There is necessity to take features of object so as to conclude identified object is targeted object or not. There are several feature extraction algorithms get developed for pedestrian detection like SIFT [2], HOG[3], haar wavelet [1] etc.

Image classification aims to categorised detected object are positive or negative i.e. if detected object is pedestrian then it is positive otherwise negative by comparing it with various standard datasets. There are different classifiers are used for pedestrian detection like SVM[1], Neural Network, Adaboost etc.

\begin{tabular}{|c|}
\hline Preprocessing \\
\hline Feature Extraction \\
\hline Object Classification \\
\hline Verification/ Refinement \\
\hline Tracking \\
\hline Pedestrian or non pedestrian \\
\hline
\end{tabular}

Fig. 1 Work flow of pedestrian detection system

\section{IMPLEMENTATION}

\subsection{Hardware}

The block diagram of pedestrian detection system is as shown in fig. 2. It includes Friendly Arm Board S3C2420, Regulated power supply, Display Unit, USB device, USB Camera, 
Buzzer, DC motor. DC motor is one of the components in PPS which represents speed or motion of vehicle. When pedestrian is get detected then motor gets stop to represent situation of vehicle stop or applying break. L293D is Dual HBridge Motor Driver IC is used. When any pedestrian is get detected, it alerts driver by providing alarm sound and also it stops vehicle automatically. The display unit in vehicle provides details about position of pedestrian either right or left. In this system we going use S3C2440 based microcontroller, which the current dominant microcontroller.

The system uses USB webcam which is connected to ARM9 board through USB device. The webcam draw one region before capturing the pedestrian. The webcam captures the pedestrian present in the region only in the form of frames by using Open CV library later it retrieves image pixel data. It compares the captured image with Haar features of a pedestrian image which is stored in the form of .xml file.

If the Haar features are matched with captured image buzzer will turn on as well as the controller apply break point to stop the DC motor through L293D driver IC to indicate the vehicle is in danger condition. If any pedestrian is not detected by webcam in the region, the motor will run in normal way.

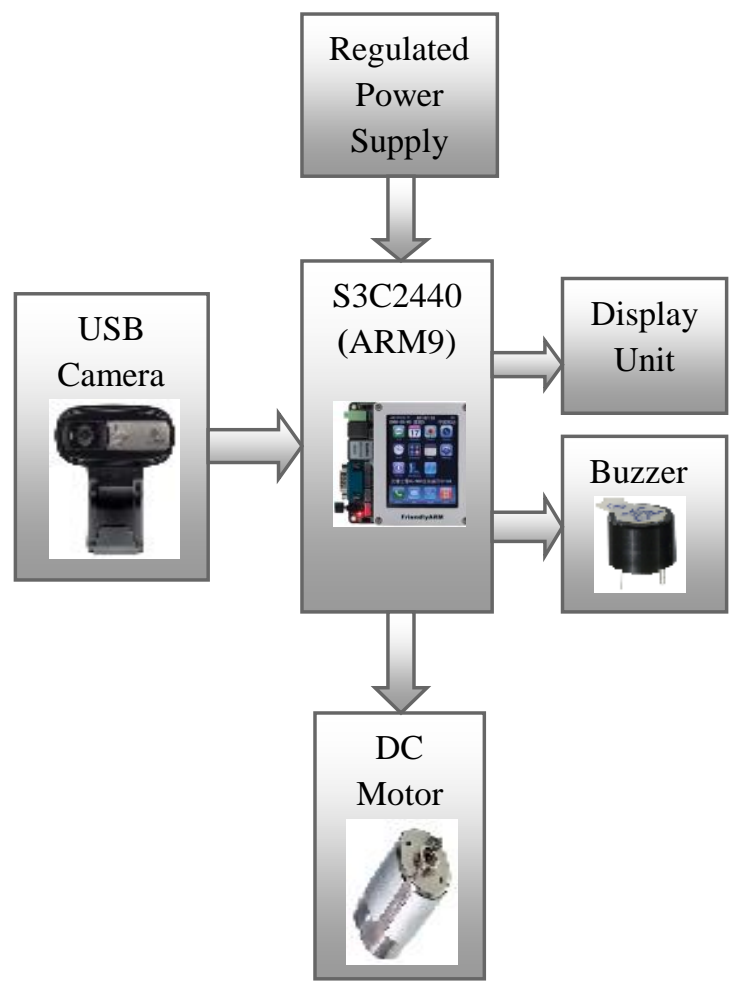

Fig. 2 Block diagram of Pedestrian protection system

\subsection{Algorithm}

Haar cascade algorithm (Viola- Jones algorithm) [3] is used for pedestrian detection. Haar cascade works in four steps, Haar Features Selection, Creating Integral Image, Adaboost Training algorithm and Cascade Classifiers. First step is Haar feature selection in which a threshold applied to sums and differences of rectangular image regions. In case of creating integral image, it computes a value at each pixel $(x, y)$ i.e. the sum of the pixel values above as well as to the left of $(x, y)$,inclusive. Computation time required for this stage is low. This can be computed within one pass through the image. The third stage is to train Adaboost algorithm, in this
AdaBoost learns a set of classifiers and also builds a voting system. The last stage is cascade classifiers; each stage in it consists of a strong classifier. So all the features are grouped into several stages where each stage has certain number of features. The work of each stage is to determine whether a given sub-window is definitely not a pedestrian or may be a pedestrian. A given sub-window is immediately discarded as not a pedestrian if it fails in any of the stages as shown in fig. 3.

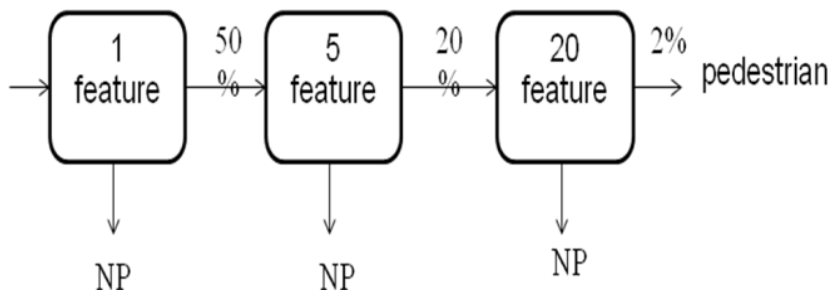

Fig.3 Cascade classifiers

\section{RESULTS}

We implemented the algorithm using Friendly ARM board S3C2440 and tested it on various datasets. From different pedestrian datasets randomly 60 images are chosen. These chosen images are with different environments like some with cluttered background, occlusion, evening time i.e. when light is low, with urban background etc. Also images which are tested includes child pedestrian, male pedestrian, female pedestrian, old pedestrian etc. Implemented pedestrian detection system is having very low false positive and low false negative rate. Pedestrian protection system is basically one of the accident prevention techniques. This system detects pedestrian, as pedestrian comes in the range of camera. And as per the situation action get perform by the processor as to apply break or not. Break applying condition is indicated by stopping dc motor. Fig. 4 shows child pedestrian is get correctly detected. Out of them some are forward facing, some are backward facing and some are side view facing all are correctly get detected. Fig. 5 shows female pedestrian with child is detected correctly. Fig. 6 shows male pedestrian with front view is detected correctly. Also there are time variations also but implemented system detects it correctly. Fig. 7 shows result of implemented system for female pedestrian with front and side view. Fig. 8 shows pedestrian with back view are get detected. Fig. 9 shows pedestrian with cluttered background is get detected correctly. Fig. 10 shows group of pedestrians get detected correctly. Fig. 11 shows group of two pedestrians are get detected correctly with different background

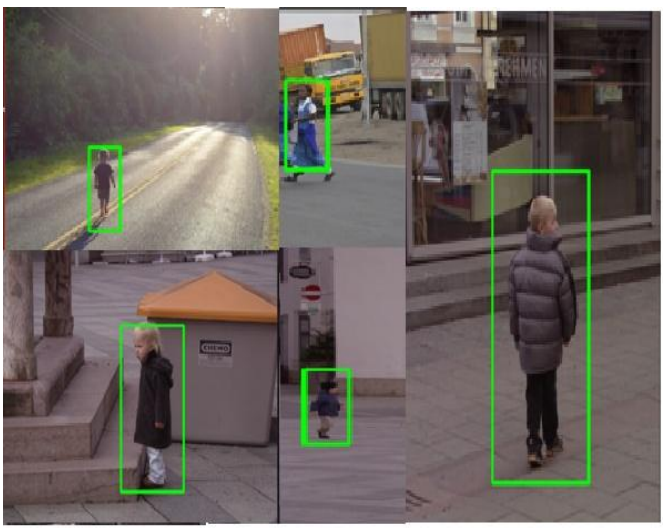

Fig. 4 Child pedestrian 


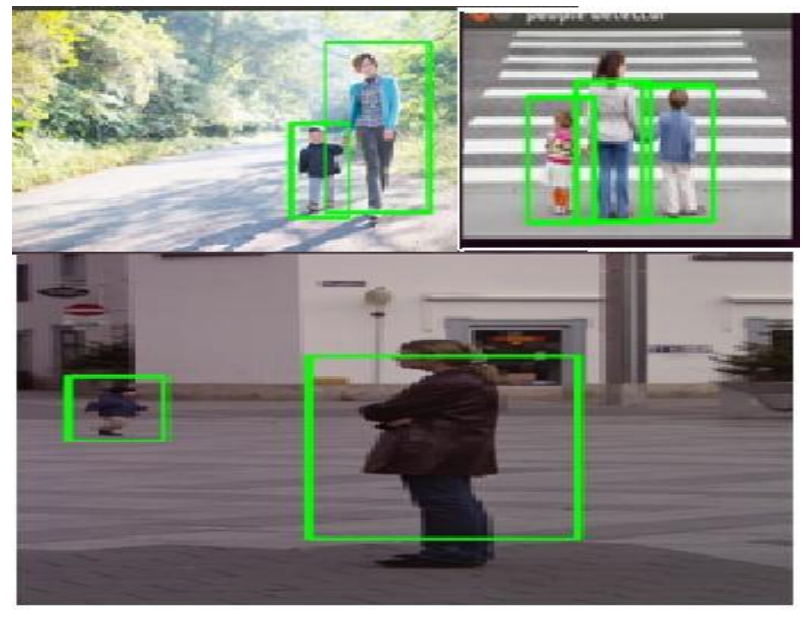

(b)

Fig. 5 Female and Child pedestrian

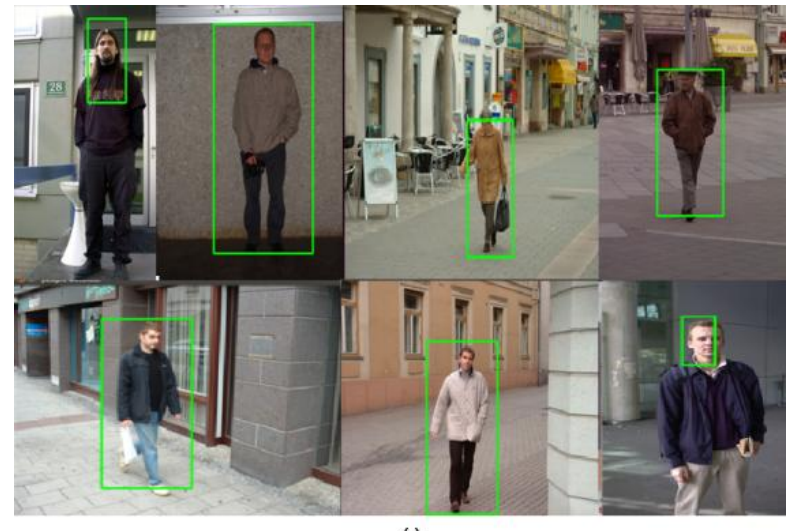

(o)

Fig. 6 male pedestrian with front view

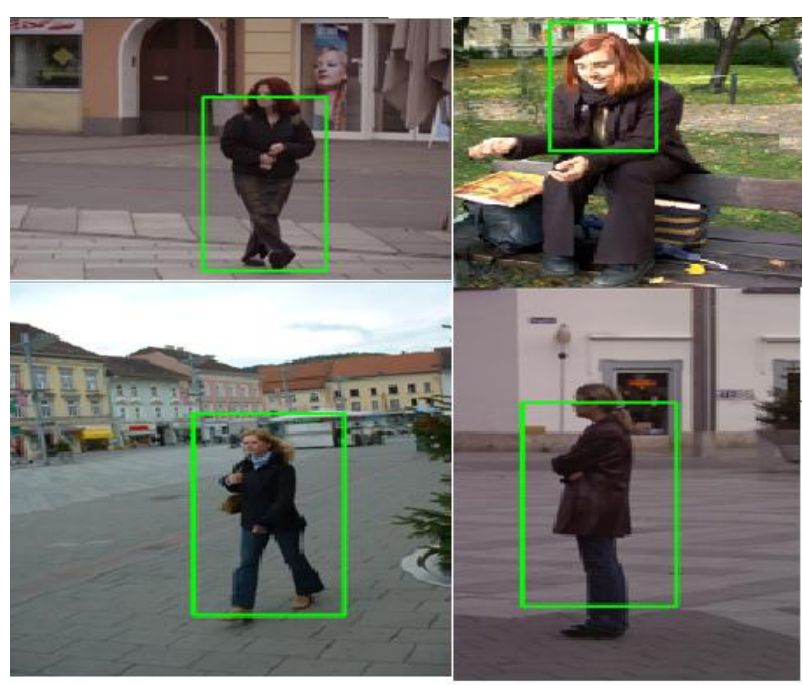

(d)

Fig. 7 Female pedestrian with front and side view

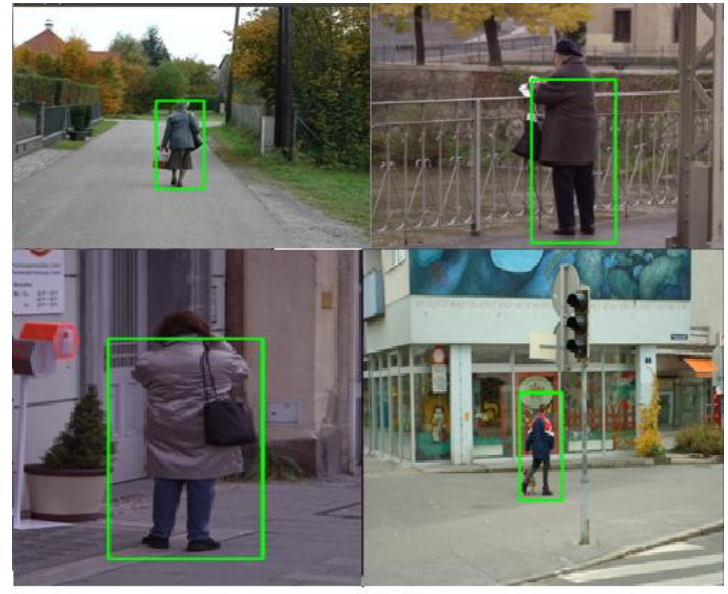

(e)

Fig. 8 Pedestrian with back view

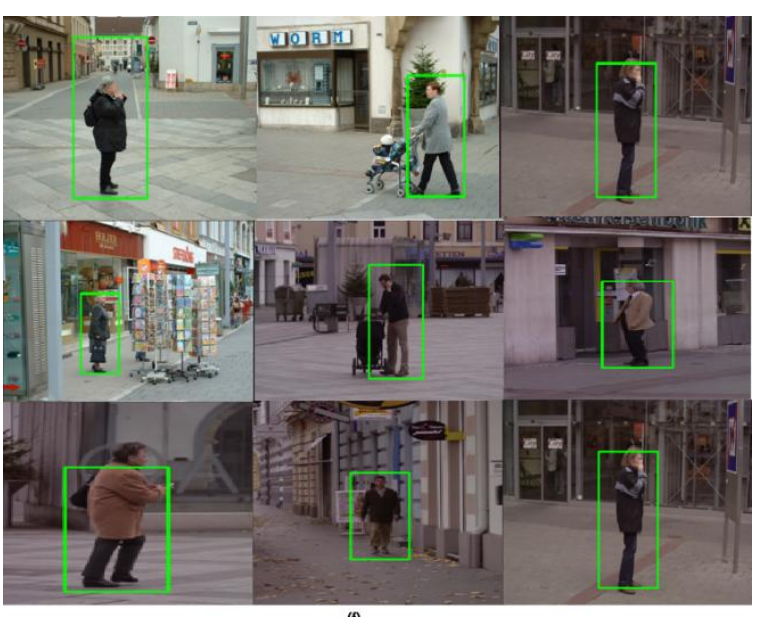

Fig. 9 Pedestrian with cluttered background

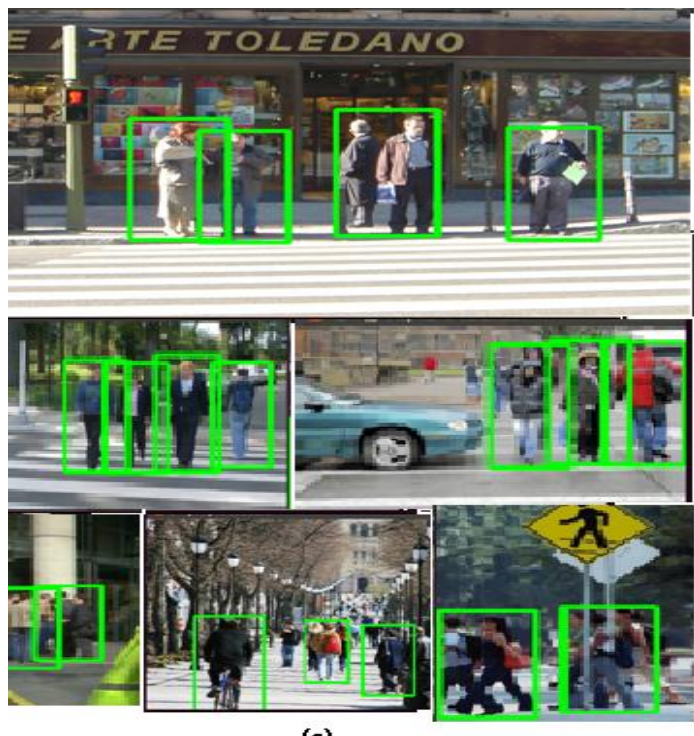

(g)

Fig. 10 Group of pedestrians 


\subsection{Confusion matrix}

Implemented algorithm is tested on 60 images. The results the same are as shown in Table 1 and also summarized as below:

Number of test images: 60

Accurate detection: 48

Accuracy: $80 \%$

Error rate: $20 \%$

Table 1 confusion matrix

\begin{tabular}{|c|c|c|c|}
\cline { 3 - 4 } \multicolumn{2}{c|}{} & \multicolumn{2}{c|}{ Predicted class } \\
\cline { 3 - 4 } \multicolumn{2}{c|}{} & Pedestrian & Non-Pedestrian \\
\hline \multirow{3}{*}{$\begin{array}{c}\text { Real } \\
\text { Class }\end{array}$} & Pedestrian & 41 & 9 \\
\cline { 2 - 4 } & $\begin{array}{c}\text { Non- } \\
\text { Pedestrian }\end{array}$ & 3 & 7 \\
\hline
\end{tabular}

Fig. 10 shows graph of accuracy vs. environmental conditions which shows that for good environmental condition i.e. having sufficient light, no rain etc conditions system has high accuracy up to $99 \%$. But for low light it is $60 \%$, for cluttered background it is around $40 \%$, for occlusion it is $20 \%$. Fig. 11 shows graph of accuracy vs. response time. As accuracy increases response time decreases. Fig. 12 shows for good environmental conditions less.

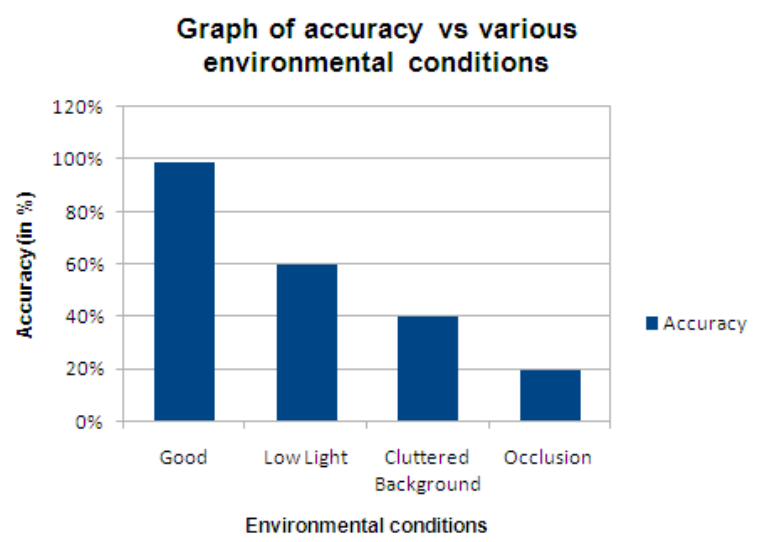

Fig. 10 Accuracy Vs environmental conditions

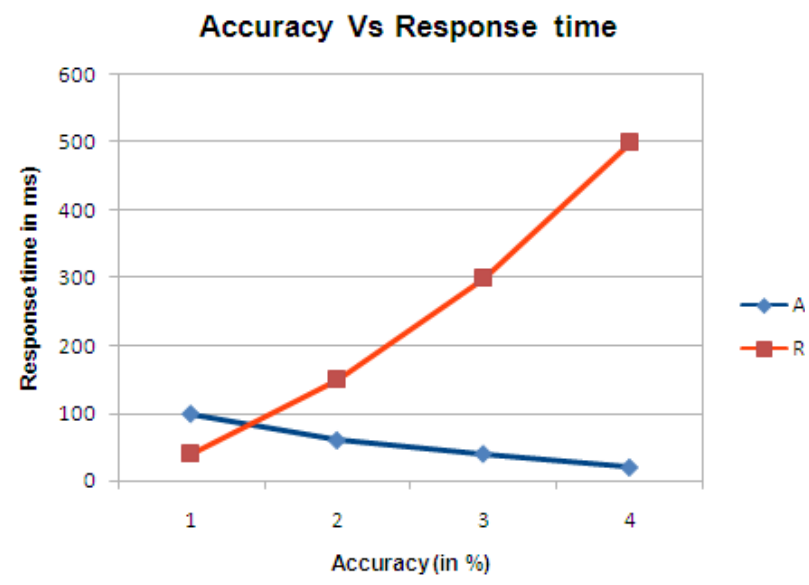

Fig. 11 Accuracy Vs Response time

\section{Environmental conditions Vs Response time}

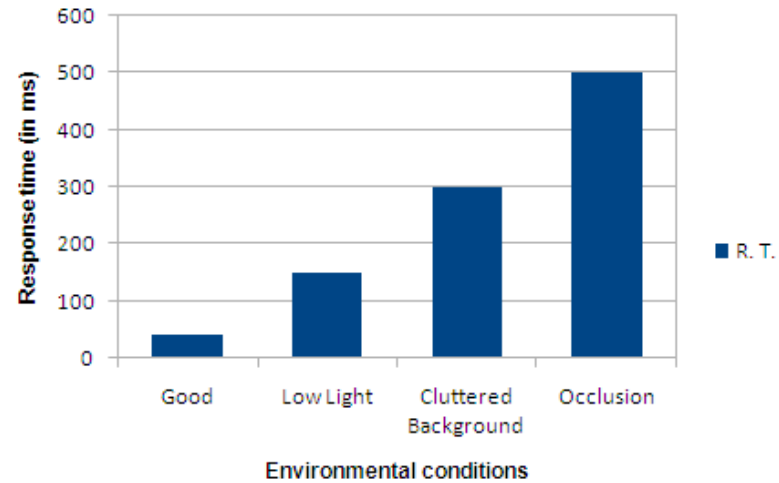

Fig. 12 Environmental conditions Vs Response time

\section{DISCUSSION AND CONCLUSION}

We implemented pedestrian protection system to avoid accidents between pedestrians and vehicles.

We use Haar cascade algorithm to detect human. Haar cascade algorithm can detect partial human also. Haar cascade algorithm has less computation time, therefore we use it. We developed it as a independent module.

We tested our system on software as well as hardware platform. In software platform we apply our algorithm on INRIA dataset. We got $80 \%$ accuracy for software platform, and $20 \%$ error rate. We also tested this system on hardware platform in real time. Results are varying depending on environmental conditions. We got $85 \%$ accuracy for good environmental conditions, and $15 \%$ error rate.

A perfect pedestrian detection system needs to work at all environmental conditions and also at night without fail. But this system should work without disturbing driver if any risk condition is not there. Cost of this system should be comparably low than that of car.

Implemented system with ARM 9 has low cost and also it has good accuracy in good environmental conditions. But still there is lot of work has to be done like systems work at night time, and also at all environmental conditions.

\section{REFERENCES}

[1] Cortes C, Vapnik V. Support vector machine[J]. Machine learning, 1995, 20(3): 273-297.

[2] Lowe, David G. (1999)," Object recognition from local scale - invariant features", proceeding of the international conference on computer vision 2.pp.11501157.

[3] P. Viola, M. Jones, Robust real-time object detection, Int.J.Comput.Vis. 57(2) (2004)137-154.

[4] P. Viola, M. Jones, and D. Snow, "Detecting Pedestrians Using Patterns of Motion and Appearance," Int'l J. Computer Vision, vol. 63, no. 2, pp. 153-161, 2005.

[5] Dalal, N. Triggs, B. : Histograms of Oriented Gradients for Human Detection, IEEE Computer Society Conference on Computer Vision and Pattern Recognition, 2005. 
[6] David Geronimo, Antonio M. Lopez, Angel D. Sappa, Member, IEEE, and Thorsten Graf, "Survey of Pedestrian Detection for Advanced Driver Assistance Systems", IEEE Transactions on pattern analysis and machine intelligence, Vol. 32, No. 7, July 2010.

[7] R. Rios-Cabrera, T. Tuytelaars, L. Van Gool ," Efficient multi-camera detection, tracking, and identification using a shared set of Haar-features", in: Proceedings of the IEEE computer society conference on computer vision and pattern recognition, pp.65-71,2011.

[8] Xianbin Cao, Zhong Wang, Pingkun Yan, Xuelong Li, "Rapid pedestrian detection in unseen scenes",Neurocomputing 74 (2011) 3343-3350.

[9] Jiang-tao Wang, De-bao Chen, Hai-yan Chen, Jing-yu Yang, "On pedestrian detection and tracking in infrared videos", Pattern Recognition Letters 33 (2012) 775-785.

[10] Lie Guo , Ping-Shu Ge , Ming-Heng Zhang, Lin-Hui Li , Yi-Bing Zhao," Pedestrian detection for intelligent transportation systems combining AdaBoost algorithm and support vector machine", Expert Systems with Applications 39 (2012) 4274-4286.

[11] Hulin Kuang, Yanwen Chong, Qingquan Li, Chunhou Zheng," MutualCascade method for pedestrian detection", Neurocomputing137(2014)127-135

[12] Chong Yan-Wen, KUANG Hu-Lin, LI Qing-Quan, Twostage pedestrian detection based on multiple features and machine learning, Acta Autom.Sin.38(3) (2012)375-581.

[13] J. Xu, D. Vazquez, A. M. Lopez, J. Marin, D. Ponsa, "Learning a Part-based Pedestrian Detector in Virtual World". In IEEE Transactions on Intelligent Transportation Systems (T-ITS), 2014.

[14] Navneet Dalal "Finding People in Images and Videos" $\mathrm{PhD}$ Thesis

[15] Datasheets and the user manuals of S3C2440. 